\title{
Cone fundamentals and CIE standards
}

\author{
Andrew Stockman
}

UCL Institute of Ophthalmology, London, UK

\section{Address}

UCL Institute of Ophthalmology, 11-43 Bath Street, London EC1V 9EL, UK

Corresponding author: Stockman, Andrew (a.stockman@ucl.ac.uk)

\begin{abstract}
A knowledge of the spectral sensitivities of the long- (L-), middle- (M-) and short- (S-) wavelength-sensitive cone types is vital for modelling human color vision and for the practical applications of color matching and color specification. After being agnostic about defining standard cone spectral sensitivities, the Commission Internationale de l' Éclairage (CIE) has sanctioned the cone spectral sensitivity estimates of Stockman \& Sharpe [1] and the associated measures of luminous efficiency $[2,3]$ as "physiologically-relevant" standards for color vision [4,5]. These can be used to model mean normal color vision at the photoreceptor level and postreceptorally. Both LMS and $X Y Z$ versions have been defined for 2-deg and 10-deg vision. Built into the standards are corrections for individual differences in macular and lens pigment densities, but individual differences in photopigment optical density and the spectral position of the cone photopigments can also be accommodated $[6,7]$. Understanding the CIE standard and its advantages is of current interest and importance.
\end{abstract}


Color perception and photopic visual function are inextricably linked to and, indeed, limited by the properties of the three cone photoceptors: the long- (L-), middle- (M-) and short- (S-) wavelength-sensitive cones. This short review covers the derivation of the recent "physiologicallyrelevant" Commission Internationale de I' Éclairage (CIE) 2006; 2015 cone spectral sensitivities and luminous efficiency functions for 2-deg and 10-deg vision [4,5] and provides background details about cone spectral sensitivities and trichromatic color matching. The CIE 2006; 2015 standards are the most secure, currently-available functions for modelling normal human color vision. The new cone spectral sensitivities are based on behavioural sensitivity measurements made in observers lacking one or both of the $\mathrm{M}$ - and L-cones, which are then used to guide the transformation of existing color matching data obtained from color normal observers. The various functions described below can be downloaded from http://www.cvrl.org.

\section{Molecular basis of univariance and trichromacy}

The photopigments that absorb light and initiate visual sensation reside in the outersegments of the cone and rod photoreceptors, which carpet the retinal surface of the eye. The photopigments are made up of a transmembrane opsin, a G protein-coupled receptor protein, made up of a sequence of amino acids, to which is bound the light-sensitive chromophore, 11-cis-retinal. The properties of our color vision depend critically on the initiating step of vision, which occurs when a photon is absorbed and isomerizes the chromophore from its 11-cis form to its all-trans form activating the opsin and triggering the phototransduction cascade [for review, see 8].

Two aspects of photoisomerization are significant for color vision. First, the absorption of a photon is an all-or-nothing event that triggers the same response regardless of photon wavelength. Consequently, once absorbed, all information about wavelength is lost, so that the response is univariant [9] or color-blind: light intensity and wavelength are confounded in the photoreceptor output. Second, the probability that a photon is absorbed depends on how closely its energy matches the optimal energy for the isomerization. This optimal energy varies with cone type because of differences in key amino acids in parts of the opsin molecule that surround the chromophore. These key amino acids modify the isomerization energy and thus shift the spectral sensitivity [10].

Having three univariant, color-blind cone types with different spectral sensitivities means that lights are represented by just three values: the three cone responses. Pairs of lights that produce the same cone responses must therefore completely match and will thus appear identical (i.e., they will be "metameric") whatever their wavelength composition. In terms of color matching, this means that the color of any "test" light can be matched by mixing together three other "primary" lights. (The matches require that the primaries be independent-in the sense that no two of the primaries can match the third, and that one of the primaries often has be added to the test light to complete the match). The intensities of the three primary lights required to match test lights as a function of test wavelength define the three color matching functions (CMFs), often defined for matches to test lights of equal energy. The upper left inset of Figure 1 illustrates a typical color matching experiment in which a test light of variable wavelength $(\lambda)$ is matched to three primaries, in this case, of 645,526 and $444 \mathrm{~nm}$. The left-hand graph shows the corresponding red, green and blue "large-field" CMFs 
measured by Stiles \& Burch [11] for fields of 10-deg in visual diameter (the other standard size is 2deg in diameter, which is known as "small-field"). For large-field color matching, observers ignore the appearance of the central area of the field. Using colorimetric notation, we refer to the largefield functions here as the $\bar{r}_{10}(\lambda), \bar{g}_{10}(\lambda)$ and $\bar{b}_{10}(\lambda)$ CMFs. The CMFs are negative when the primary in question has to be added to the test light to complete the match, when it is known as a "desaturating" primary. In the example shown in Figure 1, the 645-nm primary has been added to the test light to complete the match. Desaturating primaries are needed when the test light falls outside the volume of color space bounded by the three primaries.

[Insert Fig. 1 about here]

\section{Color matching and cone fundamentals}

Color matches are matches made at the cone level that depend on the spectral sensitivities of the L-, $\mathrm{M}$ - and S-cones. These spectral sensitivities are known as the fundamental CMFs, and in colorimetric notation as $\bar{T}(\lambda), \bar{m}(\lambda)$ and $\bar{s}(\lambda)$. The color-matching primary "lights" that underlie the three fundamental CMFs are imaginary lights that uniquely stimulate each of the three cones. Note that the fundamental cone spectral sensitivities express the sensitivity of the cones in terms of the light entering the eye and are thus different from the sensitivities expressed in term of the light captured by the cones themselves, primarily because of spectral filtering by the lens and macular pigment.

All sets of CMFs, whether for real or imaginary primaries, must be a linear transformation of these fundamental CMFs - as indicated by the bidirectional arrows in Figure 1. The upper right-hand graph shows the CIE 2006; 2015 large-field $\bar{l}_{10}(\lambda), \bar{m}_{10}(\lambda)$ and $\bar{s}_{10}(\lambda)$ CMFs and the lower righthand graph the large-field $\bar{x}_{10}(\lambda), \bar{y}_{10}(\lambda)$ and $\bar{z}_{10}(\lambda)$ CMFs. The red arrows are the linear transformations defined in the CIE standard: arrow $[\mathrm{A}]$ corresponds to Equation (3), below, and arrow [B] to Equation (5). The $\bar{x}(\lambda), \bar{y}(\lambda)$ and $\bar{z}(\lambda)$ CMFs were an invention of the CIE in 1931 that used imaginary primaries to form a color volume that was larger than the volume of real lights. Thus, real lights always map onto positive values of $\bar{x}(\lambda), \bar{y}(\lambda)$ and $\bar{z}(\lambda)$ [as they must also do, of course, for $\bar{l}(\lambda), \bar{m}(\lambda)$ and $\bar{s}(\lambda)$ ]. For these linear transformations to be valid, color matching must be linear and additive. Tests of linearity and additivity are embodied in Grassmann's Laws, which have been tested extensively and hold well [12-15].

Until the report of technical committee TC 1-36 in 2006, the CIE had been reluctant to sanction the fundamental CMFs, preferring instead to define color matches as $\bar{r}(\lambda), \bar{g}(\lambda)$ and $\bar{b}(\lambda)$ or $\bar{x}(\lambda)$, $\bar{y}(\lambda)$ and $\bar{z}(\lambda)$.

The standardization of the cone fundamentals requires the definition of linear transformation from a known set of CMFs, such as $\bar{r}(\lambda), \bar{g}(\lambda)$ and $\bar{b}(\lambda)$, to $\bar{I}(\lambda), \bar{m}(\lambda)$ and $\bar{s}(\lambda)$, thus:

$$
\left(\begin{array}{ccc}
\bar{I}_{R} & \bar{I}_{G} & \bar{l}_{B} \\
\bar{m}_{R} & \bar{m}_{G} & \bar{m}_{B} \\
0 & \bar{s}_{G} & \bar{s}_{B}
\end{array}\right)\left(\begin{array}{l}
\bar{r}(\lambda) \\
\bar{g}(\lambda) \\
\bar{b}(\lambda)
\end{array}\right)=\left(\begin{array}{c}
\bar{l}(\lambda) \\
\bar{m}(\lambda) \\
\bar{s}(\lambda)
\end{array}\right),
$$


where $\bar{I}_{R}, \bar{I}_{G}$ and $\bar{I}_{B}$ are, respectively, the L-cone sensitivities to the R, G and B primary lights, and similarly $\bar{m}_{R}, \bar{m}_{G}$ and $\bar{m}_{B}$ and $\bar{s}_{R}, \bar{s}_{G}$ and $\bar{s}_{B}$ are the analogous $\mathrm{M}$ - and $\mathrm{S}$-cone sensitivities. (Since the S-cones are insensitive in the long-wavelength part of the spectrum, it can be assumed that $\bar{s}_{R}$ is zero.) Given that we need only define the relative shapes of $\bar{I}(\lambda), \bar{m}(\lambda)$ and $\bar{s}(\lambda)$, this simplifies to:

$$
\left(\begin{array}{ccc}
T_{R} / \bar{T}_{B} & \bar{T}_{G} / \bar{T}_{B} & 1 \\
\bar{m}_{R} / \bar{m}_{B} & \bar{m}_{G} / \bar{m}_{B} & 1 \\
0 & \bar{s}_{G} / \bar{s}_{B} & 1
\end{array}\right)\left(\begin{array}{l}
\bar{r}(\lambda) \\
\bar{g}(\lambda) \\
\bar{b}(\lambda)
\end{array}\right)=\left(\begin{array}{c}
k_{l} \bar{T}(\lambda) \\
k_{m} \bar{m}(\lambda) \\
k_{s} \bar{s}(\lambda)
\end{array}\right),
$$

where the absolute values of $k_{l}, k_{m}$, and $k_{s}$ remain unknown, but are typically chosen to scale the three functions in some way; for example, so that $k_{l} \bar{I}(\lambda), k_{m} \bar{m}(\lambda)$ and $k_{s} \bar{s}(\lambda)$ peak at unity. To specify the cone fundamentals, therefore, we need at a minimum to know the relative spectral sensitivities of the cones to the $R, G$ and $B$ primaries (or $X, Y, Z$ primaries).

A common approach to recovering the unknowns in the above equations has been to measure the three cone spectral sensitivities, $\bar{l}(\lambda), \bar{m}(\lambda)$ and $\bar{s}(\lambda)$, directly. However, such measurements are complicated by the overlap of the three cone spectral sensitivities across the visible spectrum (see Figure 2). To measure the cone sensitivities separately requires the use of either special conditions of adaptation [e.g., 16,17,18 ] or color deficient observers who lack one or two of the normal cone types [e.g., 1,19]. Stockman \& Sharpe [1] used spectral sensitivity data measured in nine protanopes, who lack L-cones, twenty-two deuteranopes, who lack M-cones, and three S-cone monochromats, who lack both $\mathrm{M}$ - and L-cones $[20,21]$ to determine the unknowns in Equation (2). Importantly, all their observers had been genotyped, and the male deuteranopes and protanopes were chosen so that they had only a single opsin gene on their $X$ chromosome (and therefore had only one longer wavelength cone photopigment). Consequently, the cone photopigments in their observers were all known.

Another critical issue in defining the cone fundamentals is the choice of the $\bar{r}(\lambda), \bar{g}(\lambda)$ and $\bar{b}(\lambda)$ CMFs from which $\bar{l}(\lambda), \bar{m}(\lambda)$ and $\bar{s}(\lambda)$ should be linearly transformed. In principle, any set should suffice, but in practice the available data sets vary considerably in quality. In particular, the widely-used CIE 1931 2-deg CMFs [22] are entirely unsuitable, because they were reconstructed from relative color matching data [23,24] using the flawed $1924 \mathrm{CIE} V(\lambda)$ function [25]. Not only is such a reconstruction unnecessary and speculative, since CMFs can be measured directly, but the 1924 function also significantly underestimates luminance at short wavelengths [see 6]. The 10-deg CMFs of Stiles \& Burch [11] were chosen for the new CIE standards because they are the best and most comprehensive set of directly-measured CMFs; and unlike the related CIE 1964 10-deg CMFs have not been modified by the CIE [26]. The transformation matrix from the Stiles and Burch 10-deg $\bar{r}_{10}(\lambda), \bar{g}_{10}(\lambda)$ and $\bar{b}_{10}(\lambda)$ CMFs [11] to the three 10-deg cone fundamentals, $\bar{T}_{10}(\lambda), \bar{m}_{10}(\lambda)$ and $\bar{s}_{10}(\lambda) \mathrm{CMFs}$ is [4]:

$$
\left(\begin{array}{ccc}
2.846201 & 11.092490 & 1 \\
0.168926 & 8.265895 & 1 \\
0 & 0.010600 & 1
\end{array}\right)\left(\begin{array}{l}
\bar{r}_{10}(\lambda) \\
\bar{g}_{10}(\lambda) \\
\bar{b}_{10}(\lambda)
\end{array}\right)=\left(\begin{array}{c}
\bar{T}_{10}(\lambda) \\
\bar{m}_{10}(\lambda) \\
\bar{s}_{10}(\lambda)
\end{array}\right) .
$$


The CIE 2-deg cone spectral sensitivities are based on the same transformation, but the cone fundamentals are adjusted to macular and photopigment optical densities appropriate for a 2-deg target field [for details see 1]. The 2-deg functions are shown as logarithmic sensitivities in Figure 2 and the 10-deg functions as linear sensitivities in the upper right-hand graph of Figure 1 (both red, green and blue lines).

[Insert Fig. 2 about here]

The CIE 2- and 10-deg cone fundamentals [4] proposed by Stockman \& Sharpe [1] are arguably the most secure estimates of the mean human cone spectral sensitivities available for modelling human color vision being based on observers of known genotype. They follow a long history of cone spectral sensitivity estimates, the first plausible estimates of which were obtained in the $19^{\text {th }}$ century by König \& Dieterici [27] (shown as symbols in Figure 2). Notable estimates since then include those by Bouma [28], Judd [29,30], Wyszecki \& Stiles [31], Vos \& Walraven [32], Vos [33], Estévez [34], Vos, Estévez \& Walraven [35], and Stockman, MacLeod \& Johnson [36]. Until recently, the estimates by Smith \& Pokorny [19] have been widely used in science and vision research as a de facto standard. However, they were based on the flawed CIE 1931 CMFs that were then corrected by Judd [37] and then by Vos [33] to improve their performance at short wavelengths. Unfortunately, because the corrections that were applied were arbitrarily restricted to wavelengths shorter than $460 \mathrm{~nm}$, the Smith \& Pokorny cone fundamentals are implausible in that spectral region. Figure 2 shows the Smith \& Pokorny estimates as dashed lines and for historical context the estimates by König \& Dieterici as symbols. For the L- and M-cone fundamentals, the discrepancies between the more modern fundamentals are found mainly at shorter wavelengths where the Smith \& Pokorny functions are flawed; the discrepancies between the S-cone fundamentals are more extensive. The effects of such discrepancies on color prediction have become more important in recent years because of the use of lights with narrow-band spectral components.

\section{Cone fundamentals and luminance}

Photometry and colorimetry were artificially linked in the CIE 1931 standard because $V(\lambda)$ was used to reconstruct the $1931 \mathrm{CMFs}$ (see above). Linking $V(\lambda)$ [or $\bar{y}(\lambda)]$ to the cone fundamentals (but not using it to define them) remains convenient and useful not just for photometry (e.g., for defining $\mathrm{cd} / \mathrm{m}^{2}$ or trolands) but also for modelling postreceptoral color vision. Specifically, it is generally assumed that the postreceptoral "luminance" pathway, upon which visual functions such as motion perception and visual acuity depend, is mediated by a linear combination of the L- and M-cones with a spectral sensitivity of $V(\lambda)$ [e.g., 38]. To provide a $V(\lambda)$ function consistent with their new cone fundamentals, Sharpe et al. [2,3] measured luminous efficiency in 40 genotyped observers. From these measurements, they proposed mean $V(\lambda)$ functions for 2-deg and 10-deg vision that were linear combinations of the Stockman \& Sharpe (CIE) L- and M-cone spectral sensitivities. These functions, $\bar{y}(\lambda)$ and $\bar{y}_{10}(\lambda)$ for 2- and 10-deg vision, respectively, are defined in Equations (4) and (5), below, and form part of the CIE 2006; 2015 standard [4,5].

It should be recognised that unlike cone spectral sensitivity functions luminous efficiency or $V(\lambda)$ functions change dramatically with chromatic adaptation [e.g., 39]. Moreover, the relative contributions of the $\mathrm{L}$ - and $\mathrm{M}$-cones to luminance and thus to the shape of $V(\lambda)$ varies considerably 
across observers [e.g., 2]. For critical tasks, $V(\lambda)$ should be determined individually for each observer and for each experimental condition.

\section{Cone fundamentals and $\mathrm{XYZ}$}

By making a few further simple assumptions, the cone fundamentals, $\bar{l}(\lambda), \bar{m}(\lambda)$ and $\bar{s}(\lambda)$, can be linearly transformed to the familiar colorimetric variants: $\bar{x}(\lambda), \bar{y}(\lambda)$ and $\bar{z}(\lambda)$-a form still in common use in applied areas of research [5]. The new derivations of $\bar{y}(\lambda)$ and $\bar{y}_{10}(\lambda)$ were introduced in the previous section. $\bar{z}(\lambda)$ and $\bar{z}_{10}(\lambda)$ are simply scaled versions of $\bar{s}(\lambda)$ and $\bar{s}_{10}(\lambda)$, respectively, while $\bar{x}(\lambda)$ and $\bar{x}_{10}(\lambda)$ were chosen for consistency with the earlier CIE CMFs.

The 2-deg transformation is given by:

$$
\left(\begin{array}{ccc}
1.94735469 & -1.41445123 & 0.36476327 \\
0.68990272 & 0.34832189 & 0 \\
0 & 0 & 1.93485343
\end{array}\right)\left(\begin{array}{c}
\bar{T}(\lambda) \\
\bar{m}(\lambda) \\
\bar{s}(\lambda)
\end{array}\right)=\left(\begin{array}{c}
\bar{x}(\lambda) \\
\bar{y}(\lambda) \\
\bar{z}(\lambda)
\end{array}\right)
$$

where $\bar{l}(\lambda), \bar{m}(\lambda)$ and $\bar{s}(\lambda)$ are the CIE 2006 2-deg cone fundamentals and the 10-deg transformation is given by:

$$
\left(\begin{array}{ccc}
1.93986443 & -1.34664359 & 0.43044935 \\
0.69283932 & 0.34967567 & 0 \\
0 & 0 & 2.14687945
\end{array}\right)\left(\begin{array}{c}
\bar{I}_{10}(\lambda) \\
\bar{m}_{10}(\lambda) \\
\bar{s}_{10}(\lambda)
\end{array}\right)=\left(\begin{array}{c}
\bar{x}_{10}(\lambda) \\
\bar{y}_{10}(\lambda) \\
\bar{z}_{10}(\lambda)
\end{array}\right)
$$

where $\bar{l}_{10}(\lambda), \bar{m}_{10}(\lambda)$ and $\bar{s}_{10}(\lambda)$ are the CIE 2006 10-deg cone fundamentals.

[Insert Fig. 3 about here]

Figure 3 shows the spectrum locus plotted in four frequently-used types of chromaticity diagrams all of which are projective transformations of the CIE 2006; 2015 2-deg CMFs. The transformations are achieved by normalizing the CMFs with respect either to their sum or to luminance. Chromaticity diagrams help to visualize variations in chromaticity separately from variations in intensity or luminance. Panel $[A]$ shows the $I(\lambda), m(\lambda)$ chromaticity coordinates; Panel [B] the $r(\lambda), g(\lambda)$ chromaticity coordinates; Panel [C] the $x(\lambda), y(\lambda)$ chromaticity coordinates; and Panel [D] the MacLeod-Boynton chromaticity coordinates $I_{\mathrm{MB}}(\lambda), s_{\mathrm{MB}}(\lambda)$. Each panel shows the chromaticity coordinates of the spectrum locus as the white solid line with selected, labelled wavelengths highlighted by the solid circles. Because of the normalizations, only two of the three coordinates need to be plotted. Although the four projective transformations appear strikingly different, they reflect the same underlying color matches.

\section{Individual differences}

The CIE standards are useful for modelling color vision for observers with mean normal cone and mean normal $V(\lambda)$ spectral sensitivities. But users of the standards should be aware that individual 
differences will affect the predictions for individual observers. Individual differences occur in the density of the pigment in the lens, in the density of macular pigment at the fovea, and in the axial optical density of the photopigment in the photoreceptor. As well as differing between individuals, the last two vary with retinal eccentricity, and should be adjusted when predicting the cone spectral sensitivities for target sizes and eccentricities different from the standards. Adjustments for macular and lens pigments densities are incorporated within the CIE standard [4]. How to adjust for variations in photopigment optical density is described in Stockman \& Sharpe [6].

Variations in the spectral positions of the L- and M-cone photopigments are also common because of hybrid (mixed) L- and M-cone photopigment opsin genes, which are fusion genes produced by intragenic crossing over and thus contain the coding sequences from both $\mathrm{L}$ - and $\mathrm{M}$-cone pigment genes [for review, see 40,41]. Both in vitro [42,43] and in vivo [e.g., 20,44] measurements of the absorbance spectrum peaks of the hybrid pigments reveal a wide range of possible anomalous pigments lying between the normal L- and M-cone pigments [see Table 1 of 45]. In addition, smaller shifts occur within the normal population, because of different polymorphisms (commonly occurring allelic differences) of the $\mathrm{M}$ - and L-cone photopigment opsin genes. The most frequently observed polymorphic-induced shift (c. $3 \mathrm{~nm}$ ) occurs in the L-cone photopigment when alanine replaces serine at position 180 of the L photopigment opsin gene. The CIE L-cone fundamental incorporates a mix of these two polymorphisms [1]. How to adjust for variations in photopigment spectral position is also described in Stockman \& Sharpe [6].

\section{Acknowledgements}

The preparation of this manuscript was supported by grants from the Biotechnology and Biological Sciences Research Council (BB/M01858X/1 and BB/R019487/1). The original measurements on which the CIE standards are based would not have been possible without the efforts of Ted Sharpe. I am grateful to Thomas Smart for helpful comments.

\section{References and recommended reading}

1. Stockman A, Sharpe LT: Spectral sensitivities of the middle- and long-wavelength sensitive cones derived from measurements in observers of known genotype. Vision Research 2000, 40:1711-1737.

** The original derivation of the cone fundamentals that form the basis of the CIE physiologicallyrelevant standards.

2. Sharpe $L T$, Stockman A, Jagla $W$, Jägle $H$ : A luminous efficiency function, $V^{*}(\lambda)$, for daylight adaptation. Journal of Vision 2005, 5:948-968.

3. Sharpe $L T$, Stockman $A$, Jagla $W$, Jägle $H$ : A luminous efficiency function, $V^{*}{ }_{D 65}(\lambda)$, for daylight adaptation: a correction. Color Research \& Application 2011, 36:42-46. 
* The final derivation of the new $V(\lambda)$ functions with corrections for chromatic adaptation that were not included in the original derivation [2].

4. CIE: Fundamental chromaticity diagram with physiological axes - Part 1. Technical Report 170-1. Vienna: Central Bureau of the Commission Internationale de l' Éclairage; 2006.

*Part 1 of the CIE report with details of the physiologically-relevant standards originally defined in Reference [1].

5. CIE: Fundamental chromaticity diagram with physiological axes - Part 2. Spectral luminous efficiency functions and chromaticity diagrams, vol CIE 170-2:2015. Vienna: Central Bureau of the Commission Internationale de l' Éclairage; 2015.

*Part 2 of the $\mathrm{CIE}$ report with details of luminous efficiency and chromaticity diagrams.

6. Stockman A, Sharpe LT: Cone spectral sensitivities and color matching. In Color vision: From Genes to Perception. Edited by Gegenfurtner K, Sharpe LT: Cambridge University Press; 1999:53-87.

**Basics of cone spectral sensitivities and color matching with details of how to adjust the cone spectral sensitivities for individual differences.

7. Brainard DH, Stockman A: Colorimetry. In The Optical Society of America Handbook of Optics, 3rd edition, Volume III: Vision and Vision Optics. Edited by Bass M, DeCusatis C, Enoch J, Lakshminarayanan V, Li G, Macdonald C, Mahajan V, van Stryland E: McGraw Hill; 2010:10.11-10.56.

8. Pugh EN, Jr., Nikonov S, Lamb TD: Molecular mechanisms of vertebrate photoreceptor light adaptation. Current Opinion in Neurobiology 1999, 9:410-418.

**Excellent introduction to phototransduction and light adaptation.

9. Mitchell DE, Rushton WAH: Visual pigments in dichromats. Vision Research 1971, 11:1033-1043.

10. Deeb SS: The molecular basis of variation in human color vision. Clinical genetics 2005, 67:369377.

11. Stiles WS, Burch JM: NPL colour-matching investigation: Final report (1958). Optica Acta 1959, 6:1-26.

**Remarkable work measuring the 10-deg color matching functions.

12. Grassmann H: Zur Theorie der Farbenmischung Annalen der Physik und Chemie 1853, 165:69-84.

13. Brindley GS: Physiology of the Retina and the Visual Pathway edn 2nd. Baltimore: Williams and Wilkins; 1970.

14. Wyszecki G: Color matching and color-difference matching. Journal of the Optical Society America 1972, 62:117-128. 
15. Krantz DH: Color measurement and color theory: I. Representation theorem for Grassmann structures. Journal of Mathematical Psychology 1975, 12:283-303.

16. Stiles WS: Mechanisms of Colour Vision. London: Academic Press; 1978.

17. Stockman A, Mollon JD: The spectral sensitivities of the middle- and long-wavelength cones: an extension of the two-colour threshold technique of W S. Stiles. Perception 1986, 15:729754.

18. Stockman A, MacLeod DIA, Vivien JA: Isolation of the middle- and long-wavelength sensitive cones in normal trichromats. Journal of the Optical Society of America A 1993, 10:24712490.

19. Smith VC, Pokorny J: Spectral sensitivity of the foveal cone photopigments between $\mathbf{4 0 0}$ and 500 nm. Vision Research 1975, 15:161-171.

*Much used cone spectral sensitivity estimates.

20. Sharpe LT, Stockman A, Jägle H, Knau H, Klausen G, Reitner A, Nathans J: Red, green, and redgreen hybrid photopigments in the human retina: correlations between deduced protein sequences and psychophysically-measured spectral sensitivities. Journal of Neuroscience 1998, 18:10053-10069.

**Psychophysical measurements of normal and hybrid L- and M-cone photopigments in obsrevers of known genotype.

21. Sharpe LT, Stockman A, Jägle H, Knau H, Nathans J: L, M and L-M hybrid cone photopigments in man: deriving $\lambda_{\max }$ from flicker photometric spectral sensitivities. Vision Research 1999, 39:3513-3525.

22. CIE: Commission Internationale de l'Éclairage Proceedings, 1931. Cambridge: Cambridge University Press; 1932.

23. Wright WD: A re-determination of the trichromatic coefficients of the spectral colours. Transactions of the Optical Society 1928-29, 30:141-164.

24. Guild J: The colorimetric properties of the spectrum. Philosophical Transactions of the Royal Society of London. Series A 1931, 230:149-187.

25. CIE: Commission Internationale de l'Éclairage Proceedings, 1924. Cambridge: Cambridge University Press; 1926.

26. CIE: Proceedings. Vienna Session, 1963, Vol. B (Committee Report E-1.4.1). Paris: Bureau Central de la CIE; 1964.

27. König A, Dieterici C: Die Grundempfindungen und ihre Intensitäts-Vertheilung im Spectrum. Sitzungsberichte Akademie der Wissenschaften in Berlin 1886, 1886:805-829. 
28. Bouma PJ: Mathematical relationship between the colour vision system of trichromats and dichromats. Physica 1942, 9:773-784.

29. Judd DB: Standard Response Functions for Protanopic and Deuteranopic Vision. Journal of the Optical Society of America 1945, 35:199-221.

30. Judd DB: Standard response functions for protanopic and deuteranopic vision. Journal of the Optical Society of America 1949, 39:505.

31. Wyszecki G, Stiles WS: Color Science: concepts and methods, quantitative data and formulae edn 1st. New York: Wiley; 1967.

**The bible of Color Science superceded by an even more extensive $2^{\text {nd }}$ edition in 1982.

32. Vos JJ, Walraven PL: On the derivation of the foveal receptor primaries. Vision Research 1971, 11:799-818.

33. Vos JJ: Colorimetric and photometric properties of a 2-deg fundamental observer. Color Research and Application 1978, 3:125-128.

34. Estévez O: On the fundamental database of normal and dichromatic color vision. Edited by: Amsterdam University; 1979.

35. Vos JJ, Estévez O, Walraven PL: Improved color fundamentals offer a new view on photometric additivity. Vision Research 1990, 30:936-943.

36. Stockman A, MacLeod DIA, Johnson NE: Spectral sensitivities of the human cones. Journal of the Optical Society of America A 1993, 10:2491-2521.

37. Judd DB: Report of U.S. Secretariat Committee on Colorimetry and Artificial Daylight. In Proceedings of the Twelfth Session of the CIE, Stockholm, edn Technical Committee No. 7. Edited by: Bureau Central de la Commission Internationale de l'Éclairage; 1951:1-60. vol 1.]

38. Lennie P, Pokorny J, Smith VC: Luminance. Journal of the Optical Society of America A 1993, 10:1283-1293.

39. Stockman A, Jägle $H$, Pirzer M, Sharpe LT: The dependence of luminous efficiency on chromatic adaptation. Journal of Vision 2008, 8:1.1-26.

40. Sharpe LT, Stockman A, Jägle H, Nathans J: Opsin genes, cone photopigments, color vision and colorblindness. In Color vision: From Genes to Perception. Edited by Gegenfurtner K, Sharpe LT: Cambridge University Press; 1999:3-51.

**Good introduction to the molecular genetics of normal and defective human color vision.

41. Neitz J, Neitz M: The genetics of normal and defective color vision. Vision Research 2011 , 51:633-651.

**Review of the genetics of normal and defective color vision with some consideration of the retinal circuitry underlying color vision. 
42. Asenjo AB, Rim J, Oprian DD: Molecular determinants of human red/green color discrimination. Neuron 1994, 12:1131-1138.

43. Merbs SL, Nathans J: Absorption spectra of the hybrid pigments responsible for anomalous color vision. Science 1992, 258:464-466.

44. Neitz M, Neitz J, Jacobs GH: Genetic basis of photopigment variations in human dichromats. Vision Research 1995, 35:2095-2103.

45. Stockman A, Sharpe LT, Merbs S, Nathans J: Spectral sensitivities of human cone visual pigments determined in vivo and in vitro. In Vertebrate phototransduction and the visual cycle, Part B. Methods in Enzymology, Vol. 316. Edited by Palczewski K: Academic Press; 2000:626-650. vol 316.] 


\section{FIGURE LEGENDS}

\section{Figure 1.}

The upper left inset illustrates a color matching experiment, in which a monochromatic test field of wavelength, $\lambda$, is matched by a mixture of red ( $645 \mathrm{~nm})$, green ( $526 \mathrm{~nm})$ and blue (444 nm) primary lights, one of which, in this example the red, must be added to the test field to complete the match. The left-hand graph labelled RGB shows the $\bar{r}_{10}(\lambda), \bar{g}_{10}(\lambda)$ and $\bar{b}_{10}(\lambda)$ CMFs (red, green and blue lines, respectively), which give the amounts of each of the three primaries required to match monochromatic lights across the visible spectrum. These CMFs were measured using 10-deg diameter targets by Stiles \& Burch [11]. A negative sign means that that primary must be added to the target to complete the match. CMFs can be linearly transformed from one set of primaries to another and to the fundamental primaries (bi-directional arrows). The upper right-hand graph shows the CIE $\bar{l}_{10}(\lambda), \bar{m}_{10}(\lambda)$ and $\bar{s}_{10}(\lambda)$ 10-deg cone fundamental CMFs, and the lower right-hand graph shows the CIE $\bar{x}_{10}(\lambda), \bar{y}_{10}(\lambda)$ and $\bar{z}_{10}(\lambda)$ 10-deg CMFs (both red, green and blue lines, respectively). The arrows highlighted in red are the transformations that are defined as part of the CIE standard (see text).

\section{Figure 2.}

Comparisons between estimates of the 2-deg L-, M- and S-cone fundamentals by Stockman \& Sharpe [1], which are also the CIE 2006; 2015 standards (solid colored lines), Smith \& Pokorny [19] (dashed lines) and König \& Dieterici [27] (symbols).

\section{Figure 3.}

Chromaticity coordinates of the spectrum locus (solid white lines) based on the CIE 2006; 2015 2-deg standards with selected wavelengths (solid circles) labelled in the diagrams. Panel $[A]: I(\lambda)$, $m(\lambda)$, where $\bar{I}(\lambda)=\bar{I}(\lambda) /(\bar{I}(\lambda)+\bar{m}(\lambda)+\bar{s}(\lambda))$ and $\bar{m}(\lambda)=\bar{m}(\lambda) /(\bar{l}(\lambda)+\bar{m}(\lambda)+\bar{s}(\lambda))$. Panel [B]: $r(\lambda)$, $g(\lambda)$ where $r(\lambda)=\bar{r}(\lambda) /(\bar{r}(\lambda)+\bar{g}(\lambda)+\bar{b}(\lambda))$ and $g(\lambda)=\bar{g}(\lambda) /(\bar{r}(\lambda)+\bar{g}(\lambda)+\bar{b}(\lambda))$ for primaries of 645 , 526 and $444 \mathrm{~nm}$. Panel $[C]: x(\lambda), y(\lambda)$ chromaticity coordinates where $x(\lambda)=\bar{x}(\lambda) /(\bar{x}(\lambda)+\bar{y}(\lambda)+\bar{z}(\lambda))$ and $y(\lambda)=\bar{y}(\lambda) /(\bar{x}(\lambda)+\bar{y}(\lambda)+\bar{z}(\lambda))$. Panel [D] shows the MacLeodBoynton chromaticity coordinates, where $I_{M B}(\lambda)=0.6899 \bar{I}(\lambda) / \bar{y}(\lambda)$ and $s_{M B}(\lambda)=0.0372 \bar{s}(\lambda) / V(\lambda)$. 


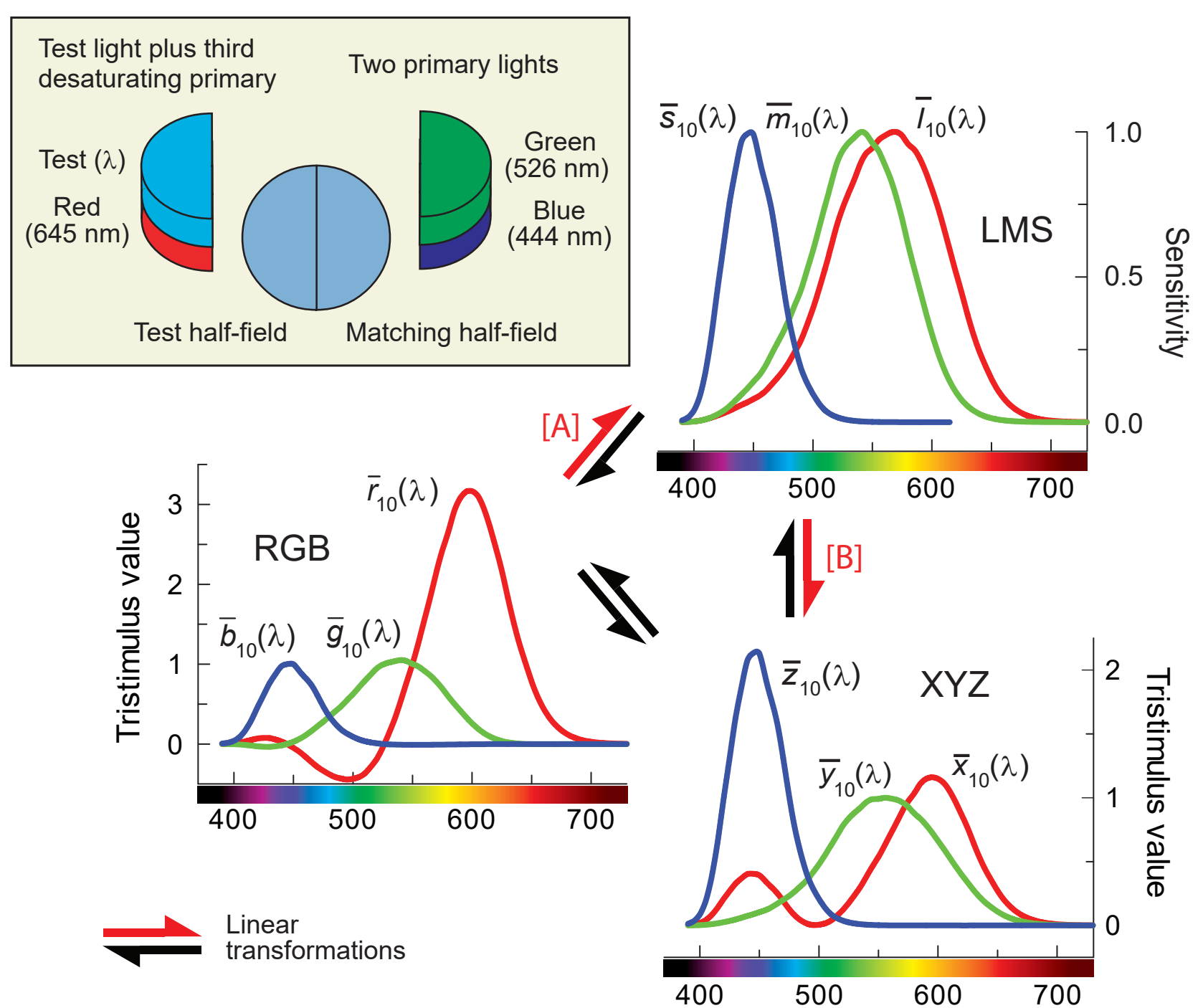

Wavelength (nm) 
FIGURE 2

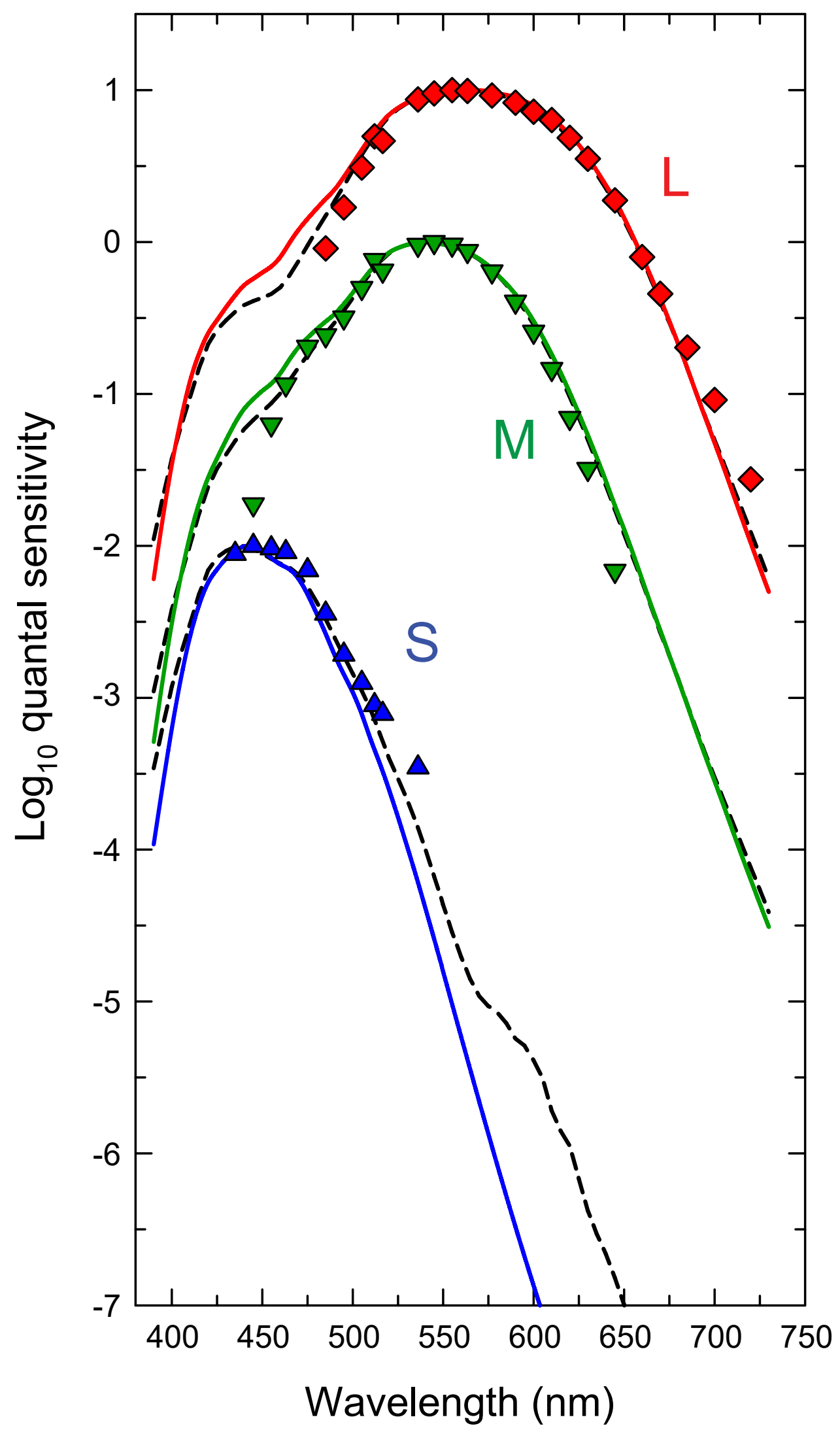


FIGURE 3
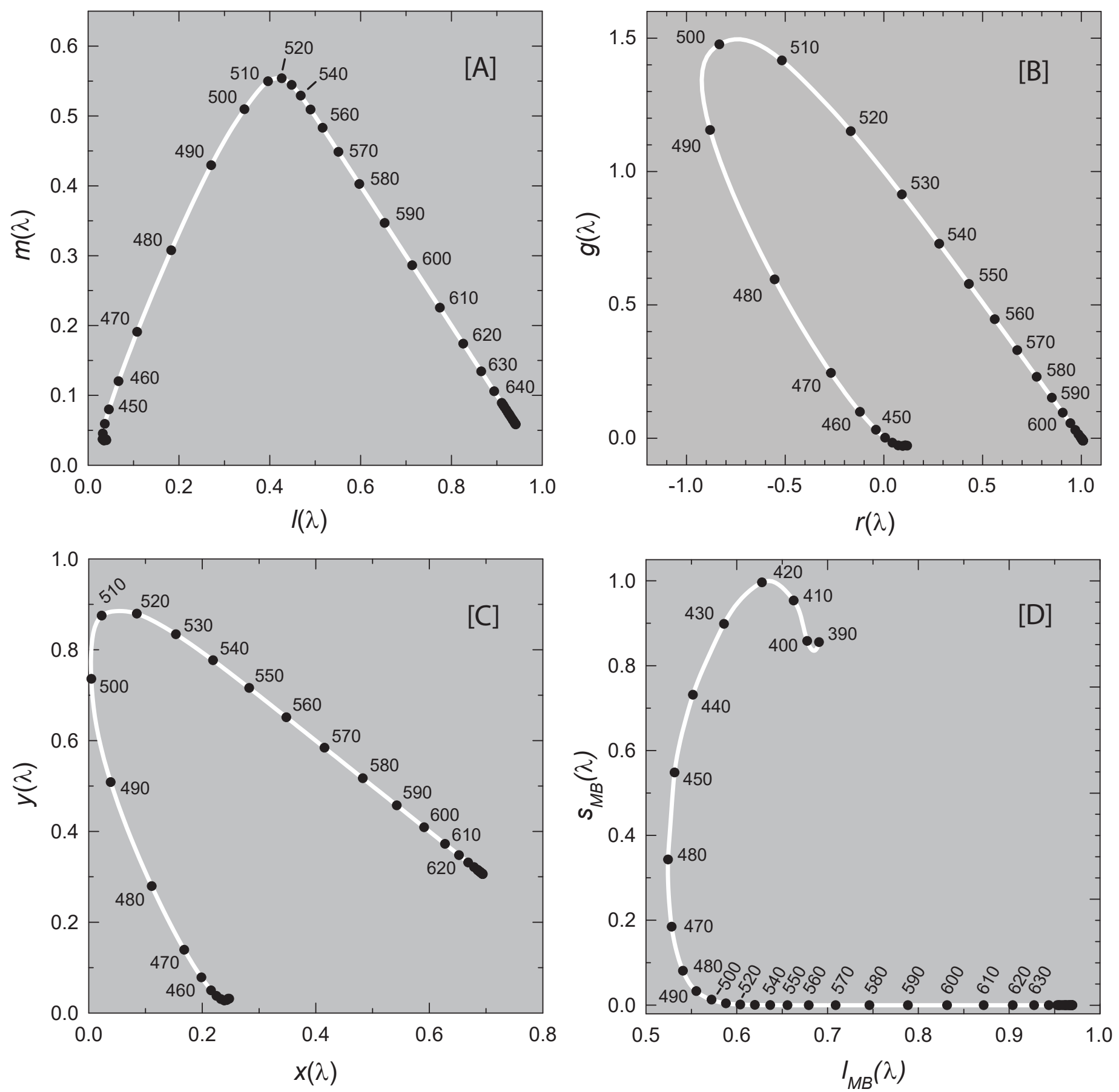